\title{
Resección bronquial en manga toracoscópica videoasistida uniportal, factible y reproducible: reporte de caso
}

\author{
Sleeve resection uniportal video assisted thoracoscopic surgery, feasible and \\ reproducible: case report
}

\author{
Juan A. Berrios-Mejía ${ }^{1 *}$, Miguel Martínez-Arias ${ }^{1}$, Ulises Loyola-Gracia', Tatzari Martínez-Coria ${ }^{1}$ y \\ María E. Marmolejo-Torres ${ }^{2}$ \\ ${ }^{1}$ Servicio de Neumología y Cirugía de Tórax, Centro Medico Instituto de Seguridad Social del Estado de México y Municipios, Toluca, Estado de \\ México; ${ }^{2}$ Servicio de Neumología, Instituto Nacional de Enfermedades Respiratorias, Ciudad de México. México
}

\section{Resumen}

La resección bronquial en manga se emplea en tumores pulmonares que invaden estructuras centrales con el objetivo de preservar tejido pulmonar funcional. El abordaje VATS uniportal es una técnica novel compleja y desafiante, que ofrece los beneficios de la mínima invasión sin incrementar los riesgos. Presentamos el caso de un paciente de 41 años con un cuadro de tos con hemoptisis por lesión exofítica del bronquio principal derecho confirmada por broncoscopia, con diagnóstico de tumor neuroendocrino de tipo 1 (tumor carcinoide), que fue resecado por completo circunferencialmente, con reconstrucción término-terminal. La evolución posoperatoria fue satisfactoria. El seguimiento se realiza en consulta externa.

Palabras clave: Broncoplastia. Cirugía toracoscópica uniportal. Resección bronquial en manga.

\begin{abstract}
Sleeve resection is used in central lung cancer and the objective is to preserve normal lung tissues. Uniportal VATS approach is a complex and challenging new technique that offers the benefits of minimal invasion, without increasing the risks. We presented a 41-year-old male patient, with history of cough with hemoptysis due to an exophytic lesion of the right main bronchus. Diagnosis of neuroendocrine tumor type 1 (carcinoid tumor) was confirmed with bronchoscopy. Complete circumferential resection was performed, with terminal-terminal anastomosis and reconstruction. Satisfactory postoperative evolution. In post-surgical follow-up.
\end{abstract}

Key words: Broncoplasty. Uniportal thoracoscopic surgery. Bronchial sleeve resection.

\section{Introducción}

La resección bronquial en manga (RBM) o broncoplastia es la resección circunferencial de una porción del bronquio, con su posterior reconstrucción. Generalmente se acompaña de una lobectomía. El objetivo del procedimiento es la preservación de tejido pulmonar funcional'.

\footnotetext{
Correspondencia:

*Juan A. Berrios-Mejía

Av. Baja Velocidad, km 57.5

Carr. Méx./Tol. 284

Col. San Jerónimo Chicahualco

Fecha de recepción: 04-02-2019

C.P. 52140, Metepec, Edo. de México, México

E-mail: juanalbertoberrios@gmail.com

Cir Cir. 2020;88(4):514-518

Contents available at PubMed www.cirugiaycirujanos.com 0009-7411/@ 2019 Academia Mexicana de Cirugía. Publicado por Permanyer. Este es un artículo open access bajo la licencia CC BY-NC-ND (http://creativecommons.org/licenses/by-nc-nd/4.0/).
} 
Inicialmente se indicó en los pacientes con tumores centrales de bajo grado con función pulmonar reducida, en quienes la neumonectomía representa un alto riesgo ${ }^{2}$. En la actualidad se emplea para reducir la pérdida sustancial de tejido pulmonar funcional y mejorar la calidad de vida ${ }^{3}$. Existe bastante evidencia en términos de baja morbilidad y mortalidad, así como de aumento de la sobrevida a largo plazo a favor de la RBM en comparación con la neumonectomía ${ }^{2,4,5}$.

La resección en manga toracoscópica videoasistida por puerto único (U-VATS) es una técnica VATS avanzada que requiere experiencia en la disección vascular, técnicas de sutura y anudado, y manejo de lesiones centrales de la vía aérea, pues se plantea la posibilidad de resección y reconstrucción bronquial y arterial. A pesar de lo laborioso de la técnica, se ha demostrado que es factible y segura, y que aporta los beneficios de la mínima invasión sin incrementar los riesgos ni comprometer la sobrevida ${ }^{6}$.

\section{Presentación del caso}

Paciente varón de 41 años sin antecedentes de enfermedades crónicas degenerativas, con un índice tabáquico de 25 paquetes-año, etilismo ocasional, consumo de cocaína ocasional durante 1 año, suspendido hace 5 años. Refiere tos con expectoración con rastros hemáticos de 2 años de evolución, disnea de medianos esfuerzos y dolor en el hemitórax derecho 4/10 en la escala visual análoga, ocasional. Niega fiebre y pérdida de peso. En la tomografía computarizada de tórax, en la ventana para mediastino se identificó una lesión endobronquial dependiente del bronquio principal derecho de $17.35 \times 14 \mathrm{~mm}$, con refuerzo al medio de contraste de más de $15 \mathrm{UH}$, sin adenopatías de mediastino (Fig. 1). Sus pruebas de función respiratorias fueron normales.

En la broncoscopia preoperatoria se evidencia una lesión exofítica con origen en la porción muscular del bronquio principal derecho, hipervascularizada, eritematosa, con obstrucción dinámica del $80 \%$ de la luz del bronquio principal derecho (Fig. 2). Se tomaron biopsias con fórceps, sin complicaciones. El resultado anatomopatológico reportó tumor neuroendocrino de tipo I (carcinoide).

Bajo anestesia general con intubación selectiva izquierda, con el paciente en decúbito lateral izquierdo, se realizó un abordaje toracoscópico uniportal de $3 \mathrm{~cm}$ de longitud, a nivel del quinto espacio intercostal línea axilar anterior, y se disecó preservando la vena ácigos. Se disecó en toda su extensión el
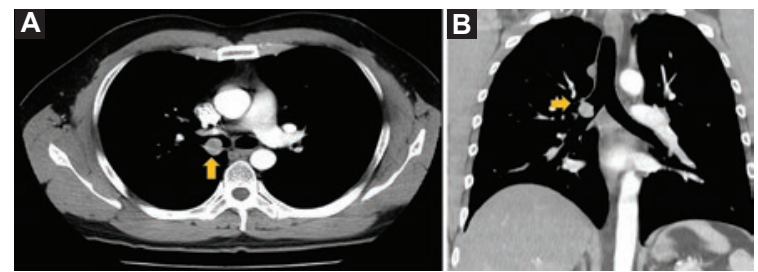

Figura 1. Tomografía computarizada de tórax contrastada. Ventana para mediastino. A: corte axial. B: corte coronal. La flecha muestra una lesión redondeada en el bronquio principal derecho.

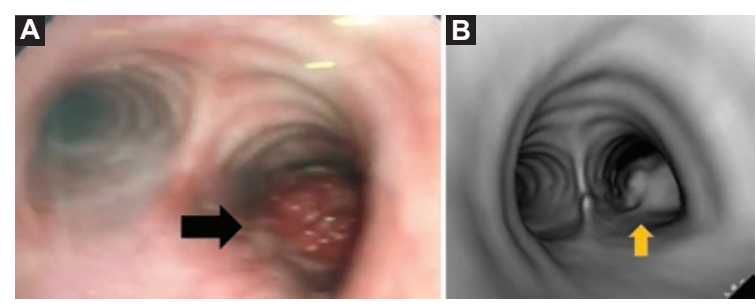

Figura 2. Imagen de lesión vista en broncoscopia en diferentes modalidades. A: videobroncoscopica flexible. B: broncoscopia virtual.
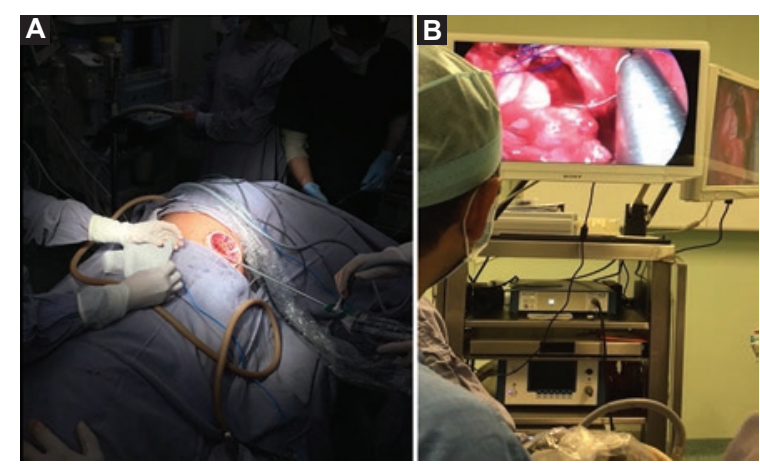

Figura 3. Técnica quirúrgica de la anastomosis término-terminal del bronquio principal derecho. A: toracoscopia videoasistida uniportal. B: sutura de la pared anterior con polipropileno 4 ceros.

bronquio principal derecho hasta los bronquios segmentarios superior e intermediario. Los márgenes de resección proximal y distal al tumor se enviaron para estudio anatomopatológico transoperatorio, que corroboró la ausencia de enfermedad. Se realizó una anastomosis término-terminal con polipropileno 4 ceros con doble aguja (Prolene ${ }^{\circledR}$, Ethicon ${ }^{\circledR}$, New Jersey, USA) con puntos continuos. Se corroboró la ausencia de fuga aérea y se colocó un drenaje de silicona de $24 \mathrm{Fr}$. El paciente cursó con adecuada evolución intrahospitalaria (solo presentó atelectasia que se resolvió con rehabilitación) y egresó al sexto día tras la cirugía (Fig. 3). 


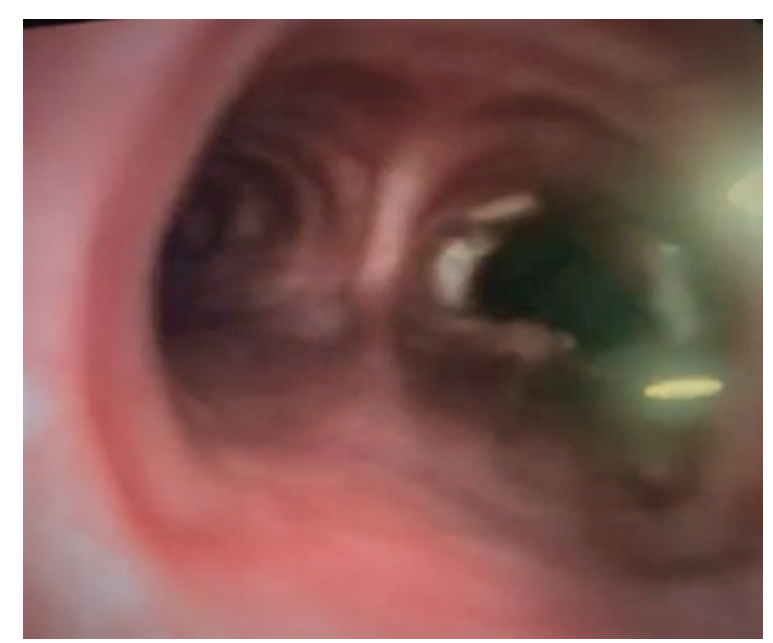

Figura 4. Videobroncoscopia flexible a los 30 días del posoperatorio.

El seguimiento se realizó en la consulta externa los días 7, 30 y 60 . Se incluyó control radiológico. Se realizó una broncoscopia el día 30 de posoperatorio, con resultados satisfactorios (Fig. 4).

\section{Discusión}

Las primeras descripciones de broncoplastias se inician cerca de 1940. Entre los pioneros se encuentra Sir Clement Price Thomas, cirujano del hospital Westminster and Brompton en Londres, quien en 1947 describió la resección circunferencial de bronquio principal por un adenoma con origen en el bronquio del lóbulo superior ${ }^{1}$. Los primeros reportes exhaustivos sobre broncoplastia corresponden a Paulson y Shaw, en 1955, al describir 18 broncoplastias en casos benignos y malignos ${ }^{3}$. En 2002, la primera descripción de una RBM toracoscópica videoasistida (VATS) fue realiza por Santambrogio, et al. ${ }^{7}$, del grupo italiano en el Hospital Policlínico de Milán. En los últimos 10 años se han descrito múltiples variaciones en la técnica, además de la evolución del abordaje U-VATS y robótico ${ }^{8-10}$.

\section{Indicación}

La RBM se ofrece en aquellos pacientes cuyas lesiones comprometen estructuras centrales (bronquio o arteria), con el fin de evitar la neumonectomía. El objetivo es preservar parénquima pulmonar funcional. La patología puede ser benigna, como una obstrucción por estenosis bronquial. En los casos de carcinoma de pulmón, la RBM debe ofrecerse cuando puede realizarse una completa resección del tumor. En pacientes con ganglios linfáticos $(\mathrm{N})$ en estaciones 1 y 2, la indicación de la cirugía es más controvertida. Otra indicación son los pacientes con pobre función pulmonar, como aquellos con enfermedad pulmonar obstructiva crónica avanzada, en los que la neumonectomía está contraindicada; en este escenario, la frecuencia de dehiscencia de la anastomosis y la mortalidad perioperatoria se incrementan ${ }^{1,3}$.

\section{Preparación preoperatoria}

La preparación del paciente incluye un adecuado estudio de imagen, como la tomografía de tórax contrastada con reconstrucción y la tomografía con emisión de positrones, para la evaluación circunferencial ante la necesidad de una neumonectomía y para una adecuada estadificación clínica. Deben realizarse las pruebas de función respiratoria de referencia (espirometría, difusión de monóxido de carbono, caminata de 6 minutos, pletismografía, gasometría), que generalmente tienden a mejorar en el posoperatorio. Se realizará una broncoscopia preoperatoria con toma de biopsia para evaluar el margen de resección, así como tratamiento preoperatorio de la obstrucción, permeabilizando el bronquio obstruido de ser necesario. Se requiere una evaluación cardiaca ante la posibilidad de una neumonectomía con sus consecuentes hipertensión pulmonar y riesgo aumentado de falla cardiaca derecha. Se seguirá un adecuado programa preoperatorio de rehabilitación y nutrición, así como consejería con psicología y manejo del dolor.

\section{Resultados}

En la literatura se reporta una mortalidad a los 30 días del $0-2.7 \% \%^{4,11-14}$, superior a la de la neumonectomía $(3.7-5.86 \%)^{2,13}$. La frecuencia de complicaciones es del 22.9-36\% $\%^{11-14}$. En la tabla 1 se desglosan la mortalidad y las complicaciones más frecuentes.

La recurrencia locorregional es del $11-22 \%$ para los pacientes operados con RBM y del $11.4-35 \%$ para los sometidos a neumonectomía ${ }^{4,13}$. La sobrevida media de los pacientes a quienes se realiza RBM es de 44.272 meses, y en aquellos con neumonectomía es de alrededor de 27.2 meses. A los 5 años la sobrevida es del $42-62.6 \%$ para la RBM y del $31-35.6 \%$ para la neumonectomía ${ }^{4,11,13,14}$.

Los pacientes con N 0-1 operados de RBM han mostrado una mayor sobrevida que los pacientes 
Tabla 1. Revisión de la mortalidad y la morbilidad en pacientes sometidos a resección bronquial en manga en los estudios que incluyen mayor número de casos

\begin{tabular}{|c|c|c|c|c|}
\hline & $\begin{array}{l}\text { Merrit, et al. }{ }^{11} \\
\quad(n=196)\end{array}$ & $\begin{array}{c}\text { Burfeind, et al. }{ }^{12} \\
\quad(n=73)\end{array}$ & $\begin{array}{c}\text { Tagawa, et al. }^{13} \\
(\mathrm{n}=151)\end{array}$ & $\begin{array}{l}\text { Yildizeli, et al. }{ }^{14} \\
\quad(n=218)\end{array}$ \\
\hline Mortalidad perioperatoria & $2 \%$ & $2.7 \%$ & $0 \%$ & $4.1 \%$ \\
\hline \multicolumn{5}{|l|}{ Complicaciones mayores } \\
\hline $\begin{array}{l}\text { Locales } \\
\text { Fistula broncopleural } \\
\text { Atelectasia lobar } \\
\text { Fuga aérea persistente (<7 días) } \\
\text { Insuficiencia respiratoria } \\
\text { Tromboembolia pulmonar } \\
\text { Empiema } \\
\text { Estenosis }\end{array}$ & $\begin{array}{c}2 \% \\
6 \% \\
10 \% \\
2.6 \% \\
1 \% \\
0 \% \\
0 \%\end{array}$ & $\begin{array}{c}1.3 \% \\
0 \% \\
1.3 \% \\
0 \% \\
0 \% \\
2.7 \% \\
9.5 \%\end{array}$ & $\begin{array}{c}4.0 \% \\
0 \% \\
0 \% \\
2.6 \% \\
0 \% \\
5.3 \% \\
0 \%\end{array}$ & $\begin{array}{c}1.8 \% \\
1.8 \% \\
2.2 \% \\
1.8 \% \\
1.37 \% \\
2.2 \% \\
1.8 \%\end{array}$ \\
\hline $\begin{array}{l}\text { Sistémicas } \\
\text { Infarto agudo de miocardio } \\
\text { Accidente vascular cerebral }\end{array}$ & $\begin{array}{c}1 \% \\
0.5 \%\end{array}$ & $\begin{array}{l}1.3 \% \\
1.3 \%\end{array}$ & $\begin{array}{c}0.66 \% \\
0 \%\end{array}$ & $\begin{array}{c}0.45 \% \\
0.9 \%\end{array}$ \\
\hline \multicolumn{5}{|l|}{ Complicaciones menores } \\
\hline $\begin{array}{l}\text { Arritmia } \\
\text { Neumonía }\end{array}$ & $\begin{array}{l}0 \% \\
9 \%\end{array}$ & $\begin{array}{c}20.5 \% \\
1.3 \%\end{array}$ & $\begin{array}{l}15.2 \% \\
13.2 \%\end{array}$ & $\begin{array}{l}1.8 \% \\
7 \% \%\end{array}$ \\
\hline
\end{tabular}

neumonectomizados (71.5 y $42.8 \%$, respectivamente). En los pacientes $\mathrm{N} 2$ no se observa diferencia estadística según el tipo de intervención ${ }^{13}$. Los estadios $\mathrm{N}$ 0-1 tienen sustancialmente una mejor sobrevida que el $\mathrm{N} 2^{14}$.

Shi, et al. ${ }^{2}$ realizaron un metaanálisis y encontraron una mortalidad perioperatoria del $2.91 \%$, frente al $5.86 \%$ en los operados de neumonectomía. La frecuencia de complicaciones fue del $32.8 \%$, mayor que en los pacientes con neumonectomía (27.6\%), pero sin diferencia estadísticamente significativa. Hubo una reducción de la recurrencia locorregional en los pacientes con RBM, con un $14.4 \%$, en comparación con el $26.06 \%$ en los neumonectomizados. La sobrevida también favoreció al grupo con RBM.

Los predictores de complicaciones en resecciones segmentarias en manga identificados fueron la edad mayor de 70 años ${ }^{11}$, el compromiso en las pruebas funcionales respiratorias (volumen espiratorio forzado en el primer segundo $<40 \%$ ), el tabaquismo, la cirugía del lado derecho, la bilobectomía, el carcinoma epidermoide y ser $\mathrm{N} 2$ positivo ${ }^{14}$. En el grupo con quimioterapia de inducción (neoadyuvancia) presentó mayor riesgo de complicaciones anastomóticas ${ }^{11}$.

Zhou, et al..$^{15}$ evaluaron el abordaje toracoscópico con técnica de tres puertos y encontraron que es factible realizar RBM de forma segura al compararlo con el abordaje por toracotomía. Requiere un tiempo quirúrgico mayor, reduce la estancia hospitalaria, no incrementa los riesgos perioperatorios en el paciente $\mathrm{y}$ tiene similares patrones de sobrevida.
La evolución de la técnica es el abordaje uniportal toracoscópico. Gonzalez-Rivas, et al. ${ }^{16}$ se dieron a la tarea de describir y difundir la técnica para resección bronquial, arterial y carinal ${ }^{16}$. En este punto ya se está realizando una transición a la técnica robótica, demostrando su factibilidad y seguridad ${ }^{8}$. El manuscrito fue preparado de acuerdo con las pautas Surgical CAse REport (SCARE) Guidelines ${ }^{17}$.

\section{Conclusiones}

La RBM por abordaje toracoscópico videoasistido uniportal es reproducible de forma segura, sin un incremento de las complicaciones perioperatorias. Es importante la difusión y el entrenamiento de los especialistas en técnicas uniportales avanzadas.

\section{Conflicto de intereses}

Los autores declaran no tener conflictos de intereses.

\section{Financiamiento}

Recursos propios de los autores y la institución.

\section{Responsabilidades éticas}

Protección de personas y animales. Los autores declaran que para esta investigación no se han realizado experimentos en seres humanos ni en animales. 
Confidencialidad de los datos. Los autores declaran que han seguido los protocolos de su centro de trabajo sobre la publicación de datos de pacientes.

Derecho a la privacidad y consentimiento informado. Los autores han obtenido el consentimiento informado de los pacientes y/o sujetos referidos en el artículo. Este documento obra en poder del autor de correspondencia.

\section{Bibliografía}

1. Beauchamp G. Fundamentals of standard sleeve resection bronchoplasty sleeve lobectomy lung cancer. Thorac Surg Clin NA. 2018;28:285-9.

2. Shi W, Zhang W, Sun H, Shao Y. Sleeve lobectomy versus pneumonectomy for non-small cell lung cancer: a meta-analysis. World J Surg Oncol. 2012;10:1-9.

3. Deslauriers J, Tronc F, Grégoire J. History and current status of bronchoplastic surgery for lung cancer. Gen Thorac Cardiovasc Surg. 2009;57:3-9.

4. Deslauriers J, Gre J, Jacques LF, Piraux M, Goujin L, Lacasse Y, et al. General thoracic sleeve lobectomy versus pneumonectomy for lung cancer: a comparative analysis of survival and sites or recurrences. Ann Thorac Surg. 2004;77:1152-6.

5. Ferguson MK, Lehman AG. Sleeve lobectomy or pneumonectomy: optimal management strategy using decision analysis techniques. Ann Thorac Surg. 2003;76:1782-8.

6. Agasthian T. Video-assisted thoracoscopic bronchoplasty. J Vis Surg. 2017;13:12.
7. Santambrogio L, Cioffi U, De Simone M, Rosso L, Ferrero S, Giunta A. Video-assisted sleeve lobectomy for mucoepidermoid carcinoma of the left lower lobar bronchus: a case report. Chest. 2002;121:635-6.

8. Tan GJS, Poon JS, Khoo PLZ, Yoong AWH, Nardini M, Dunning J. Robotic left lower sleeve lobectomy with bronchoplasty for the removal of a carcinoid tumour. J Vis Surg. 2018;8:3-5

9. Mahtabifard A, Fuller CB, McKenna RJ. Video-assisted thoracic surgery sleeve lobectomy: a case series. Ann Thorac Surg. 2008;85:S729-32.

10. Agasthian T. Initial experience with video-assisted thoracoscopic bronchoplasty. Eur J Cardio-thoracic Surg. 2013;44:616-23.

11. Merritt RE, Mathisen DJ, Wain JC, Gaissert HA, Donahue D, Lanuti M, et al. Long-term results of sleeve lobectomy in the management of non-small cell lung carcinoma and low-grade neoplasms. Ann Thorac Surg. 2009;88:1574-82.

12. Burfeind WR, Amico TAD, Toloza EM, Wolfe WG, Harpole DH General thoracic low morbidity and mortality for bronchoplastic procedures with and without induction therapy. Ann Thorac Surg. 2005;80:418-22.

13. Tagawa T, Iwata T, Nakajima T, Suzuki H, Yoshida S, Yoshino I. Evolution of a lung-sparing strategy with sleeve lobectomy and induction therapy for non-small cell lung cancer: 20-year experience at a single institution. World J Surg. 2016;40:906-12.

14. Yildizeli B, Fadel $E$, Mussot $S$, Fabre $D$, Chataigner $O$, Dartevelle $P G$ Morbidity, mortality, and long-term survival after sleeve lobectomy for non-small cell lung cancer. Eur J Cardiothorac Surg. 2007;31:95-102.

15. Zhou S, Pei G, Han Y, Yu D, Song X, Li Y, et al. Sleeve lobectomy by video-assisted thoracic surgery versus thoracotomy for non-small cell lung cancer. J Cardiothorac Surg. 2015;10:116-200.

16. Gonzalez-Rivas D, Yang Y, Stupnik T, Sekhniaidze D, Fernandez R, Velasco $\mathrm{C}$, et al. Uniportal video-assisted thoracoscopic bronchovascular, tracheal and carinal sleeve resections. Eur J Cardiothorac Surg. 2016;49:6-16.

17. Agha RA, Fowler AJ, Saeta A, Barai I, Rajmohan S, Orgill DP, et al. The SCARE Statement: consensus-based surgical case report guidelines. Int J Surg. 2016;34:180-6. 